\title{
Integrated medical system using DICOM and HL7 standards
}

\author{
Orza Bogdan ${ }^{1}$, Cordos Alin², Vlaicu Aurel ${ }^{1}$ and Meza Serban ${ }^{1}$ \\ Technical University of Cluj Napoca ${ }^{1}$ \\ Pixeldata ${ }^{2}$ \\ Romania
}

\section{Introduction}

Informational systems and the standardization of data transfer protocols have become an important part of nowadays medical world. Software applications transformed themselves into an indispensable tool for the specialist engaged in the medical act. In this sense, the use of information and communication technology has influenced greatly the fast and reliable (also secure) transmission of medical data throughout informational systems, shortening the required processing times.

The increasing use of IT\&C in the medical world also demanded for new protocols that would facilitate data transfer (between different software application) and data storage in a common format. Likewise, several organizations have undertaken this difficult task of medical digital data standardization, among which the National Electrical Manufacturers Association (NEMA) and American College of Radiology (ACR) are the most important ones. Their efforts concentrate mostly in the development of the DICOM (Digital Imaging and Communications in Medicine) standard. It has all started in the 1970's with the increasing use of CT scanners and other image-based diagnostic devices that opened the way for large - scale deployment of software applications in the medical field and thus the need to interface machines and applications manufactured/produced by various companies was born. This translated into the need to specify a common image format for all medical imaging devices.

In 1983, ACR and NEMA delegated a committee to solve this problem and propose a standard that would allow the following:

- Data exchange concerning generated digital medical images between devices produced by different manufactures;

- The development of PACS (Picture Archiving and Communication Systems);

- The development of medical databases able to be interrogated using (geographically) distributed software applications;

In 1985 version 1.0 of the DICOM standard was published by ACR and NEMA, followed by 2 revisions in October 1986 and January 1988. Version 2.0 of the standard was issued also in 1988 and added a set of commands for displays, by introducing a new image identification scheme based on "data-elements" for a better characterization of the image parameters (NEAC, 2008). 
The last published version, 3.0, issued in 2000, contains a large number of changes and additions with respect to the previous ones. The DICOM standard facilitates medical imaging equipments inter-operability by specifying:

- A set of protocols that compliant devices must withstand;

- The commands' semantics and syntax as well as the associated information format that can be transmitted using the protocol.

In a heterogeneous system, in order to integrate medical equipments supplied by various manufactures, the use of DICOM and HL7 standards is compulsory. DICOM, as previously presented, is mainly dedicated to medical imaging, whereas HL7 (Health Level Seven) covers more general aspects of medical digital data processing and management. HL7 is used for transmitting data related to patient charts, files and other associated documents and audio recordings. The number " 7 " refers to the "application" layer, the 7 th one from the OSI (Open Systems Interconnection) model system representation (HL7, 2008).

The HL7 standard was first published in 1987 by a group of companies manufacturing medical equipment. Version 2.0 succeeded in 1988, followed by versions 2.1, 2.2, 2.3 and 2.3.1 in 1990 to 1999. In 1994 ANSI (American National Standards Institute) officially recognized it as an industry standard. Currently, version 3.0 is on its way, with a draft already released.

The main objective of the HL7 standard is to produce a set of specifications that allows free communication and exchange of data between medical software applications in order to eliminate or reduce incompatibility among different applications. To achieve this, the following measures have been proposed:

- the standard must support information exchange between systems implemented in a large variety of development environments (technical) and communication environments. Its implementation must be possible in all the major existing programming languages.

- immediate, single transaction, transfer must be available in the same time as file sharing/transfer based on multiple-transactions;

- the highest degree of standardization must be obtained when compared to the most often encountered cases of elements formatting; the standard must comply with the specific necessities of each medical field. Accordingly, the standard comprises situation specific tables, definitions and segments that can be customized (Z-segments)

- the standard must cope with variations suffered in time due to inherent technical progress and evolution;

- the standard evolution must be based on the experience already gained and on already existing and well known industrial protocols. Favors given to certain producers or companies must be avoided by all means.

- $\quad$ HL7 makes no presumptions related to the architecture of the medical informatics system, and does not try to resolve the architectural differences present in medical informatics systems. Due to this reason, HL7 cannot have a "plug and play" interface.

- a first interest of the HL7 workgroup was to use the standard as soon as possible. Once published, HL7 was voted and recognized as a standard by the American National Standards Institute (ANSI) and the Accredited Standards Organization (ASO). 
- currently, the cooperation with other standardizing organization from the medical field (ACR/NEMA DICOM, ASC X12, ASTM, IEEE/MEDIX, NCPDP, etc.) has become a priority for HL7 and focus on a better development of medical informational systems has contributed to the group's joining to the ANSI HISPP (Health Information Systems Planning Panel) process, ever since its debut, in 1992.

The two standards, DICOM and HL7, form the basis of the informational integration of software based medical processes. In November 1998, Healthcare Information and Management Systems Society (HIMSS) and Radiological Society of North America (RSNA) founded the Integrating the Healthcare Enterprise (IHE) forum, with the declared goal of helping the integration of software application from various medical fields and domains. Its main objective is to ensure that in the course of the medical act all the information necessary in the decision making and taking processes are accurate and available in time for the medical specialist. Its purpose is not to define new standards, but to promote the use of the existing ones, namely DICOM and HL7. Currently the main focus is on radiology. The DICOM and HL7 standards provide the necessary means and technology for developing software applications, while IHE supervises their adoption into real-life medical world. IHE provides support for the medical software applications users by ensuring a better access to information and eliminating, as much as possible, confusions or misunderstanding when acquiring such applications.

From the medical software application development point of view, the IHE specifications facilitate fast and safe releases of new products as well as simple mechanisms for implementing interfacing options with other, already existing ones.

\section{Using the HL7 and DICOM standards in medical informatics}

\subsection{General overview of the HL7 standards}

The HL7 standard addresses software developers and medical equipments manufactures with the declared goal of unifying the way the information present in medical units and institutions is transmitted, exchanged and/or stored, based on a common format, agreed by all the parties involved. There are other standards dedicated to the medical sector, each having a very well defined domain and focus: pharmacy, medical devices, medical imaging, and insurances. HL7 is, on this matter, dedicated to the processing and management of administrative and clinical data (HL7, 2008). The HL7 focuses on the following fields/domains:

- Patient management - admit, discharge, transfer patient (ADT);

- Queries, resources (rooms, beds, devices, etc.), patient scheduling;

- Scheduling of medical procedures, results, clinical trials;

- Financial administration;

- Medical documents;

- Medical records;

- Medical treatments;

Taking into considerations the great variety of applications involved in the process of the medical act and the requirement that these have to exchange information/data between them, it is obvious that many of such communication interfaces would greatly benefit from using a standardizes approach. The HL7 standards comes exactly to solve this problem and ease the burden of message passing and data exchange between various applications by providing a very precise structure under which this must happen. 


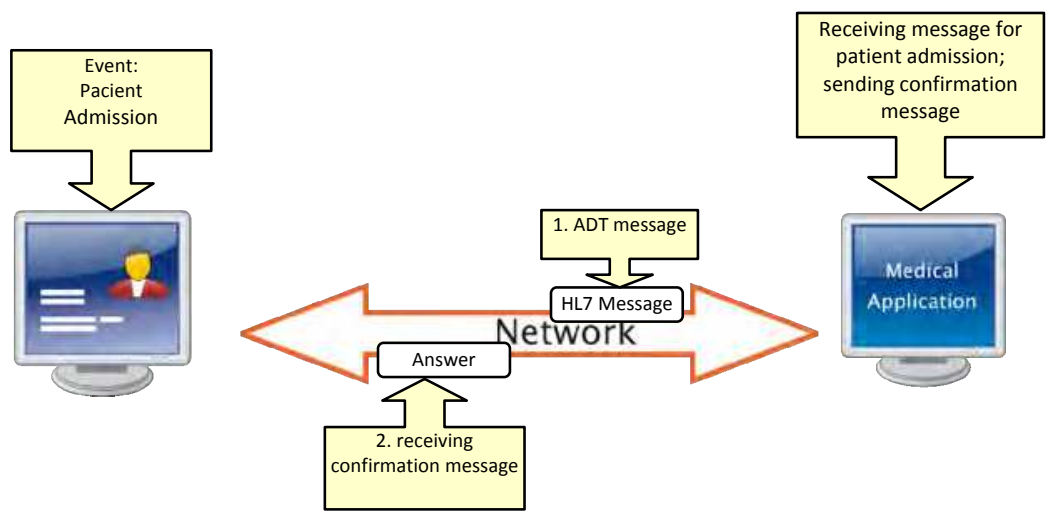

Fig. 1. Example of using HL7 messages

A medical application that uses the HL7 standard sends to another application a "HL7 type" message generated as a result of some medical event occurring in the current activity: admit patient -A01, transfer patient - A02, discharge patient - A03, etc. If the application receiving the messages also complies with HL7 regulations, then it can be certain that there will be no missing information, all information received will be interpreted the right way and a proper response will be issued back. Thus, the exchange of information made is coherent and efficient (HL7, 2008).

The HL7 standards define a number of messages that cover all activities specific to medical units. A HL7 message is made up of segments, fields, components and sub-components and is characterised by the message type (a 3 character code). Message types are organized by different domains/fields (e.g. admit, discharge, transfer, clinical trials scheduling, etc).

\subsection{HL7 scenarios}

Figure 2 presents various scenarios that make use of the HL7 standards inside a single hospital/medical unit and also among several hospitals. In a hospital, information is managed by an application generically named HIS (Hospital Information System). Using this all other activities taking place can be monitored and controlled. The application thus has different interfaces that allow communication with all the existing sub-systems, like the imaging center (RIS - Radiology Information System), financial department, human resources, pharmacy, etc. In order to connect to the "outside world" a web-based application is used. This allows reading and writing of information in the hospital data base. As it can be seen in figure 2, HL7 enables the communication among different software applications existing in the hospital, each developed by various/different companies.

For a better communication between the hospital and other different medical institutions, the HL7 standard can be used to perform data exchanges. It is enough for the informational systems in these institutions to have a HL7 compatible interface.

The HL7 standard is used for exchanging information related to patients, doctors, resources and documents between hospitals. At this level there are also data synchronization protocols that ensure data persistence among applications. By using the HL7 standard, this data exchange can be performed independently of the software solutions, operating systems 
or data base management systems running at each institution. What is important is the information contained by the HL7 message.

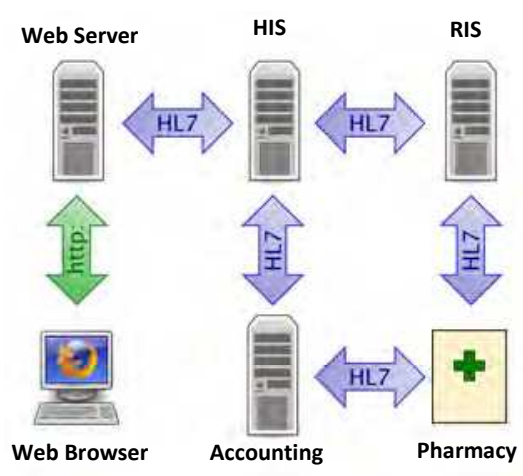

a)

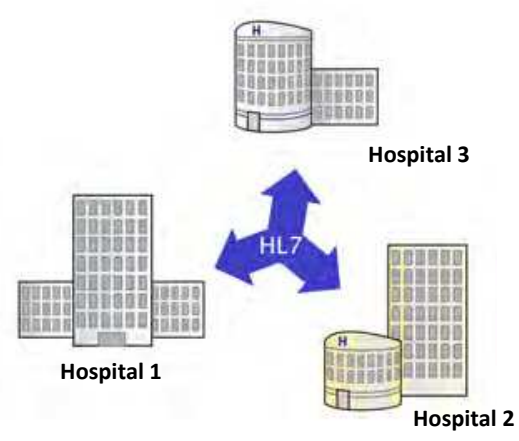

b)

Fig. 2. Example of using HL7 standard: a) inside a hospital b) between hospitals

The major benefit of standardization is represented by the informational integration. The benefits that HL7 brings to the medical system are that of having the possibility of accessing the medical information/data and process them independently on the particular solution chosen by each institution. Thus, it has no importance who the provider for a certain software application is, as long as this is compliant with the standards' specification, and it limits by no means the choices existing in acquiring other systems as well (they do not necessarily need to be developed by the same company in order to work well together).

\subsection{HL7 - Clinical Document Architecture CDA}

HL7 CDA is an XML-based standard that specifies the structure and semantics of clinical documents having in mind the exchange of such data. Previously known as PRA (Patient Record Architecture), CDA provides a model for clinical document interchanging, such as "epacris", "evolutions", etc. and makes it possible for the integration of the information technology in the medical field. CDA allows for the clinical documents to be compatible to the informatics field by using XML, HL7 RIM (Reference Information Model) and medical informatics codes but also tries to maintain a high degree of understanding and assimilation by the medical staff. The content of CDA documents can be viewed using web browsers (due to the fact that they are based on XML).

The CDA standard is based on the following concepts:

- CDA documents are based on the XML standard (Extensible Markup Language);

- The structure of the CDA documents is based on the HL7 reference model named RIM (Reference Information Model) and the HL7 data types;

- The CDA specification are very flexible and are structured on 3 levels:

○ Document level;

- Section level

- Entity level

- A CDA document is a completely defined object and can include text, images, sound recording and other multimedia components; 
HL7 CDA clinical documents contain observations and services and have the following characteristics/attributes:

- Persistency: continues to exist in an unaltered state for a well-defined period of time:

- Organization: maintained by an organization entrusted with this task;

- Possibility to certify: contains a set of information that can be certified;

- Context: sets a predefined value;

- Integrity: the authentication of a clinical document is made as a whole and is not applied to parts of the document without taking into account the whole context;

- Readability: the possibility of being read. Guaranties that the obtained CDA document can be easily viewing using a web-browser.

\subsection{The DICOM standard}

The DICOM (Digital Imaging and Communications in Medicine) standard was developed with the purpose of helping the distribution, display and storage of medical images (CT, MRI, US) in mind. The standard is more than just a file/image format, being in fact a set of standards that describe the way digital data used in medicine can be transferred, stored and displayed.

The DICOM standard has a series of advantages (Oleg S. Pianykh, 2008):

- Is a universal standard. Generally, all medical equipments acquiring medical images support this standard and communicate among them using it;

- Images are stored at a higher quality. DICOM supports:

○ JPEG compressed images: lossless JPEG compression for 8, 10, 12, 16 bits gray levels, or 24 bit color images, or lossy JPEG compression for 8, 12 bits gray levels or 24 bits color images;

- JPEG 2000 compressed images: lossless or lossy JPEG 2000 image compression for 8-16 bits gray level or 24 bits color images

- RL (run-length) compressed images

- Uncompressed images (bitmap)

- Allows the storage of additional information related to the image acquisition parameters. DICOM can store besides the actual 2D image additional information, such as: the patients 3D position, physical size of the objects present in the image, slice thickness, exposure parameters, and others. These are used for a better later processing and interpretation.

- Support for full storage of medical data. The DICOM files and messages support more than 2000 standardized attributes that maintain patient's medical data and images

- Images are acquired and stored using parameters that are device independent. Likewise, DICOM images can be processed without taking into account the actual device used in the acquisition process. 


\subsection{IHE (Integrating the Healthcare Enterprise) specifications}

IHE is an initiative dedicated to stimulating the integration of informational systems present in modern health care institutions. Its main objective is to guarantee the fact that all the information about the patient and its state necessary in the treatment and health care process are correct, accurate and available in time for the medical staff. With more than 450 billion people ensured in 27 countries, Europe represents a unique challenge for IHE (www.ihe-europe.net, 2008). IHE functions as a forum that aims at integrated all the existing informational systems present in the medical field. The IHE objectives are (O. Pianykh,2008):

- To describe the problems and their solutions concerning software application integration;

- To instrument best-practices cases and manuals for the deployment of the existing standards deployment

- To deliver an optimal support for the informational flux

- To integrate the IT-based solutions in the medical field.

IHE identifies a subset of functional components from a medical informatics system and describes its interactions using a set of transactions. The actors and the transactions described in the IHE specifications are abstractions of the medical system present in a hospital. The reason behind introducing these actors and transactions is to be able to define the interactions between the entities forming a medical informatics system.

The actor - represents the role assumed by an entity of the system. The main actors defined according to the IHE specifications are: image acquisition devices, patient registration systems, cost calculation systems for billing the performed medical procedures, medical examination management systems, image, medical reports and associated information visualization systems, image storage management and archiving systems, medical reports management systems, medical reports visualization systems, medical images printing servers (DICOM Print Server) and the financial administration systems.

Transactions are interactions between actors that transfer the required information through standards-based messages.

The integrating profiles offer a common language that healthcare professionals and vendors may use in communicating requirements for the integration of products. Integration Profiles describe real-world scenarios or specific sets of capabilities of integrated systems. An Integration Profile applies to a specified set of actors and for each actor specifies the transactions necessary to support those capabilities.

The DICOM and HL7 standards provide the necessary means and technology for the medical software applications development, while the IHE ensures the specifications needed for implementing these into the real-world. IHE specifications include the following equipments and medical software applications:

- Informatics systems that ensure patient and resource management for medical centers (HIS, RIS);

- Imaging and investigation equipments from the medical field (MR, CT, US, CR, DX, etc.);

- Software applications from the field of medical imaging (PACS, Review Stations, Reporting Systems);

- Other equipments from the medical field (Printers, Imagers, etc.). 


\section{Patient management in medical imaging centers}

Patient and resource management in a medical imaging center is provided by informatics systems generically named RIS (Radiology Information System). From the software point of view these can be classified as ERP (Enterprise Resource Planning) application.

The main functions of RIS informatics systems are: patients registration, examination scheduling, automated distribution of documents, the possibility to operate on more imaging centers in the same time, data base access management, document scanning and storing, radiological scanning library management, imaging center resource management (medical equipment, cameras, available procedures), automated cost calculation and billing associated to the medical act, patients medical insurance policy management, management of the individual procedures undertaken by each patient, patient history management, patients allergy evidence management, avoiding procedure incompatibilities (warnings).

One of the main components of such a system is the PACS server (Picture Archiving and Communication Systems). The PACS is a system (comprised of both hardware and software) meant for storing digital medical images using the DICOM standard. All PACS must be compliant with the DICOM standard. All medical image acquisition devices (CT, RMN, US) store the generated DICOM images in a PACS, from where are than later on available for display using DICOM viewers.

Communication interfaces are used for exchanging data between the RIS informatic system and adjacent software applications. They are compatible with the HL7 standard and use the TCP/IP communication protocol. All interfaces are compliant with the HIPAA (Health Insurance Portability and Accountability Act) regulations. Some of these interfaces are designed based on the IHE specifications. Figure 3 describes the connection between the RIS server and other medical informatics systems (HIMSS and RSNA, 2005).

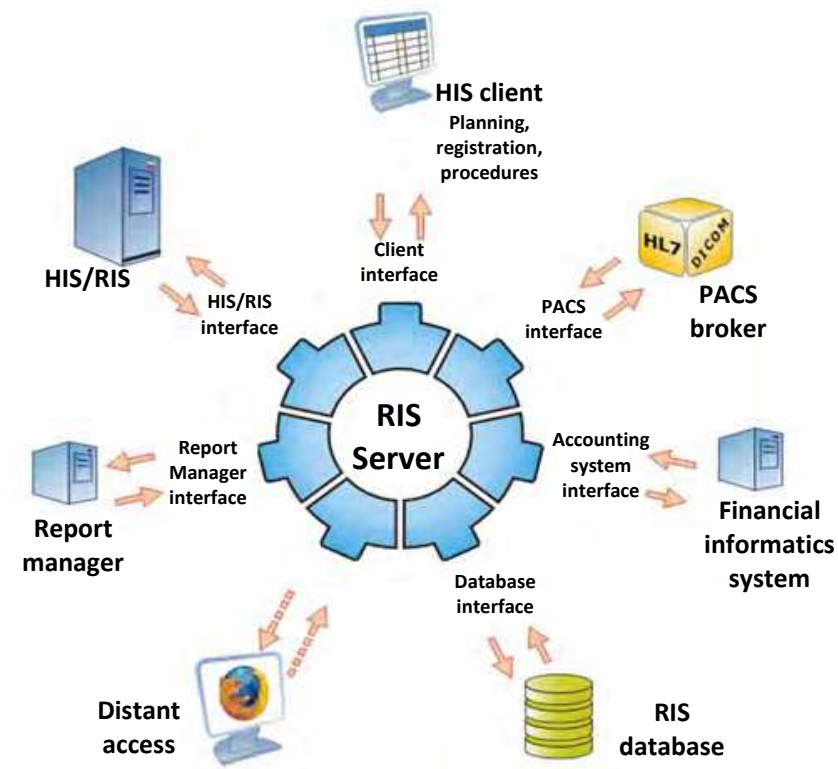

Fig. 3. Communication interfaces of the RIS server 


\subsection{The communication interface with the HIS application}

This is an interface compatible with the HL7 standard and the IHE (Integrating the Healthcare Enterprise) recommendations and is bidirectional. Through this interface information regarding the imaging center like: admission of new patients, patients relocations in beds, patient discharges, treatment changes, calls for further investigations, information regarding the medical staff, the degree of deployment of the medical resources and equipments, etc. can be exchanged. The HL7 messages exchanged by this interface are:

- Messages referring to patient admission, discharge or transfer;

- Messages referring to medical examinations

- Medical reports - for this type of action one single type of message is used, which includes the original medical rapport as well as latter additions to it;

\subsection{The communication interface between the RIS and the report management application}

This is a HL7 and IHE compatible interface and is used by the report server application to send the results for the procedures that have been made by the equipments in the imaging center to the RIS. Also through this interface the report server updates the information regarding patients and procedures. The RIS informs the report server that there are procedures that have ended and a report is required for them.

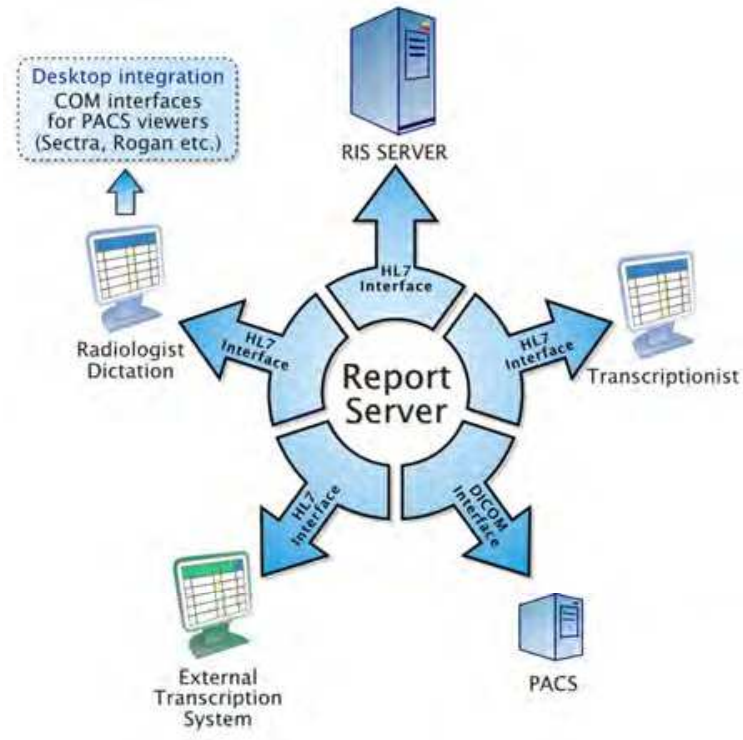

Fig. 4. System for management of medical reports

The management of reports in an imaging center is ensured by a suite of software applications made up from a server and two client applications (fig 4.). All communication between the 3 is based on HL7 messages. The three applications forming the report management system are (note that the medical report is the result of an image based investigation performed by the radiologist): 
a) The Report server - ensures the interfacing between the clients and the data base as well as the security and integrity of the data stored in the data base. The automated and manual distribution of reports is made with the help of the server application. This also maintains a permanent connection to the RIS server, updating continuously (as quickly as possible) the information referring to patients, allergies, diagnostics, procedures and the medical staff.

b) The Radiologist client - is used by the radiologist in order to record or write the medical report based on the examination of the images retrieved from the PACS server. This application can call other COM interfaces belonging to other software programs dedicated to medical images visualization (Alin Cordoş et al., 2008)

c) The Translating client - is an application dedicated to the translation of the spoken medical reports, stored as .wav files, to written form (text file). After formatting, the report is send back to the radiologist for verification and signing.

\subsection{The WEB server interface}

This is again a HL7 compatible interface destined to connect the WEB servers with the RIS. A special WEB application running inside an internet browser can receive the information from the RIS through the WEB server. Also, through this application one can inquire for a new investigation for a patient, find out available time slots in the centers' schedule, and in the same time check the status of a selected procedure.

\subsection{The RIS client communication interface}

Through this HL7 compatible interface the RIS client can perform the following tasks:

- Patient recording;

- Patient admission/discharge/transfer

- New investigation inquiry

- Manual or automatic patient scheduling

- Patient rescheduling

- Information regarding costs/prices

- Patient history

- Information regarding consumable items in the imaging center;

- Radiological film management;

\subsection{The communication interface with the PACS informatics system}

Through this interface the exchange of messages between the RIS and the PACS Broker software application is made. The PACS Broker converts HL7 messages into DICOM messages and the other way around. The following HL7 messages are used:

- Adding new patients and modifying existing patients information: ADT^A04, $^{\wedge}$ $\mathrm{ADT}^{\wedge} \mathrm{A} 08$.

- Adding / modifying medical examinations: ORM^ O01.

- Adding / modifying medical reports: ORU^ ${ }^{\wedge} 01$.. 


\subsection{The communication interface with the imaging acquisition equipment}

This interface refers to the communication between the software applications responsible for the creation, storage and management of images (medical equipment, image servers (PACS) and any electric device having a communication interface compatible with the DICOM standard) as well as those responsible for patient and resource management (RIS and/or HIS servers).

The conversion interface between the DICOM and HL7 standards is based on the requestreply principle. The entities that initiate the protocol are medical imaging equipments or PACS servers. The interface has the following characteristics:

- Works as an interface between the PACS or the medical equipment and RIS

- Allows multiple TCP/IP simultaneous connections

- Is based on the communication standards used in the medical field (DICOM, HL7)

- Implements "Modality Work list Information Model - MWLIM" from the DICOM - SOP Class UID 1.2.840.10008.5.1.4.31 standard

- Is compliant with the IHE specifications

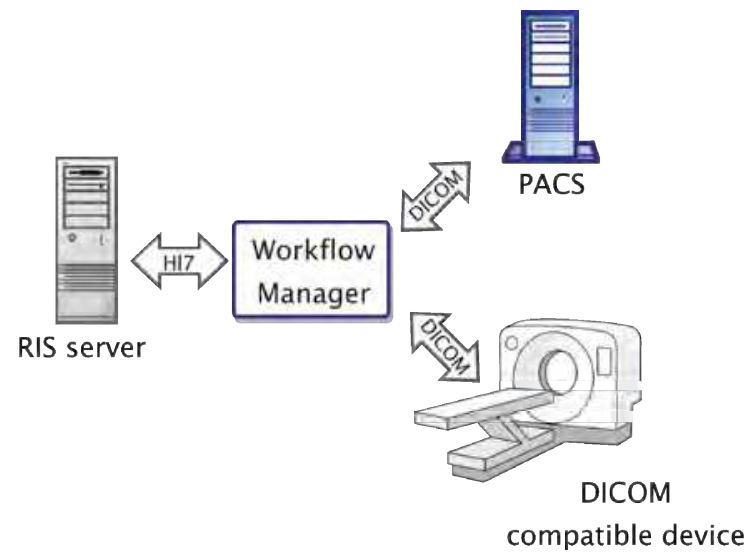

Fig. 5. Workflow manager

The interface works in the following way:

Step 1: the PACS initiates a query to the MWL - Modality Work list - this is a software application that stands between the PACS, RIS and medical imaging equipment. This is the application that informs in real time the equipment operator about the patients that must be investigated and the type of procedure to be undertaken.

Step 2: Using the filter requested by PACS, MWL initiate a HL7 type query message to the RIS server.

Step 3: Based on the answer received from the RIS server, MWL fills in all the DICOM fields requested by the PACS and sends answers to the entity that initiated the communication.

\section{Remote and distant access to information using WEB technologies}

In order to remotely access the information stored in the healthcare unit informational system a system based on web technologies has been developed. This technology was used 
because it allows the users (mostly doctors) to deploy the system even if they are not inside the health care unit.

A client/server application that is able to run inside an internet browser allows the users to access information from the imaging center like: patients, procedures, reports, images associated to certain procedures.

- The server is implemented under the form of a .dll using ISAPI (Internet Server API) and runs inside the IIS (Internet Information Services) server;

- The client is implemented using the Active $X$ concept and scripting languages such as VBScript and JavaScript

The architecture of the web application is presented in the figure below:

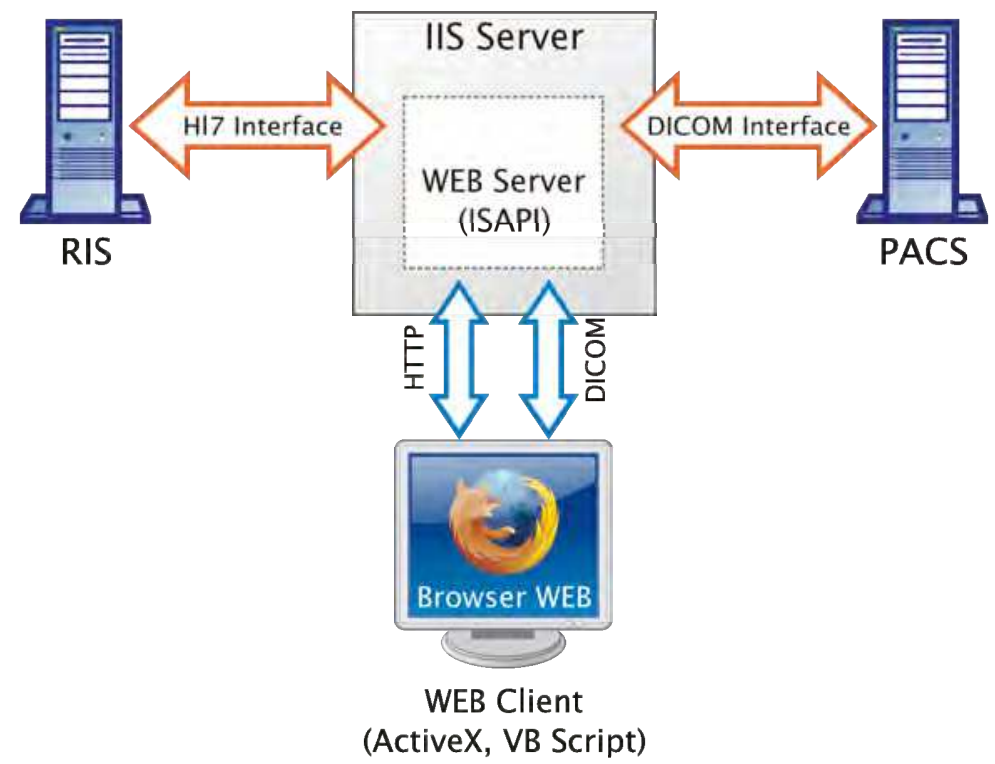

Fig. 6. Web application architecture

The servers take the requests coming from the client running in the web browser and send them to the RIS or PACS server. From the RIS server information regarding patients, medical examinations, procedures and reports are retrieved. In order to transfer the images in the shortest time possible inside the browser, the web server has an image buffer implemented. When the user asks for an image, after visualization the server stores it for a certain period of time, in case the user wants to review the image again. Moreover, to further obtain a decrease in the time necessary for an image to be available for the viewer, a JPEG compression of the images coming from the PACS server is made prior to sending them to the web client.

Usually the web servers are installed outside the "firewall" security type systems that are present in imaging centers; due to this, a careful planning and implementation of the communication interfaces is necessary. In this manner, the communication between the RIS server and the web server is made using encryption algorithms and secure transmission protocols like SSL (Secure Sockets Layer) (Alin Cordos et al., 2004). 


\section{Integrated Management System for Medical Information Using HL7 Standard - SIMIMED}

The theoretical and practical knowledge gained by the authors of this chapter in the field of medical software development were used in the PNII-D11019/2007 research project funded under the Romanian national research program scheme. The project aimed at developing a pilot HIS integrated system using the latest standards available (HL7 and DICOM), adapted to the particular case of the Romanian National Health System and compliant with the EU requirements. The SIMIMED project contains a packet of software modules that provide 4 service categories:

- The HIS integrated system: is made up from the server application and the client applications distributed by sections/departments. The system uses the 3 tier architecture and the communication between the clients and the server is based on the http/https protocol. The HIS is used for saving information in the database, client application management and database querying. The server connects to the Microsoft SQL Server database using an ADO connection and uses the web service principle loaded by the IIS server; each service is workflow - oriented. The HIS client applications offers the client a friendly and intuitive graphical user interface that allows for the user to input or retrieve data into/from the SIMIMED system. It is implemented in C\# and communicates with the server using the http/https protocol.

- The Datacenter - is a server application that manages the data stored in the common data base by all the HIS applications running in different hospitals/institutions. This stores medical data to be used by researchers. The storage center was developed based on the Microsoft SQL server technology. The HIS servers running at the medical institutions involved in the project send data to the storage center. In the data exchange process between the HIS servers and the Datacenter the HL7 together with the TCP/IP protocol are used. Due to the fact that the data stored is dedicated mainly to research purposes, no information that would allow patient identification is stored.

- The web application - is a web-client application that has/gives/allows access to the data stored in the Datacenter. It can be used by researchers, students and PhD students to study different cases classified by the Datacenter. The access is controlled by the data center administrator, who is entitled to issue access accounts based on username/password pairs.

- The AdminTool application - is a web application used for remote administration of the HIS server, and allows for quick and in time support in case of malfunctions. 


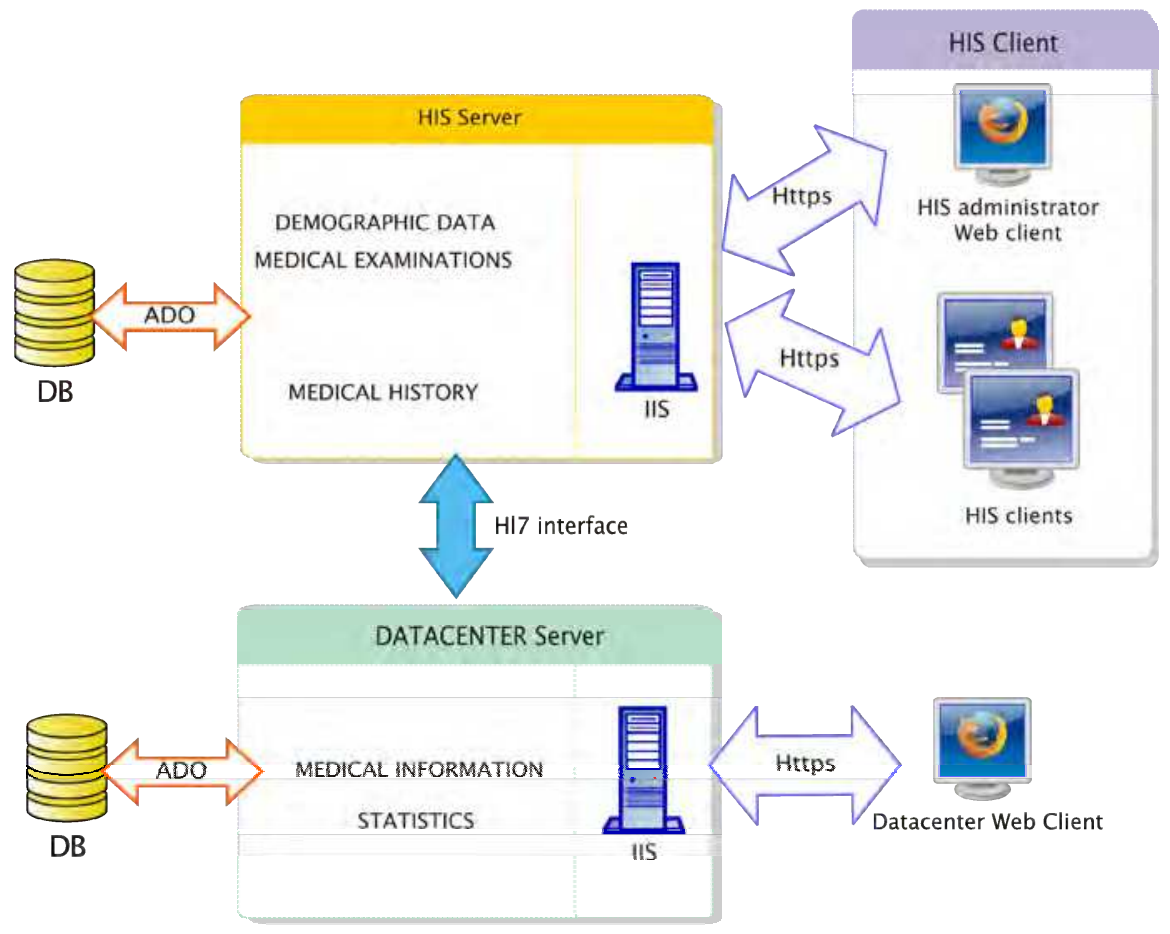

Fig. 7. SIMIMED system architecture

The SIMIMED HIS client contains five important modules: Patient Demographic, Examinations, Surgery, Patient History and Schedule.

The Patient Demographic module contains the graphical controls to add/edit/search demographic data of a patient. Using this module one can add/modify visits, document upload/download documents, add/modify transfers or other type of information (allergies, antecedents, and medical history), health insurance, and patient discharge.

The Examinations module allows the editing of information regarding examinations taken on a patient, and contains a visit list and 4 tab-controls: general clinical examinations - for general information (height, weight, general condition), examinations - for surgery procedures, specialty examinations (ex. oncology, radiology), and evolution and treatment. Data in each tab-control is loaded from the server based on the selected visit, and can be modified if the selected visit is the current one.

The Surgery module contains components regarding surgery intervention. We can edit information related to intervention date, operating physician, assistants, medical equipments or tools used and the list of surgery interventions in the selected visit.

The Patient History module contains in a single tab-control all the relevant information regarding patient visits: the list of visits, list of surgery interventions, medications, documents, allergies, transfers.

The Schedule module is used by physician to schedule meetings with patients, interventions and other events. This module contains a reminder for upcoming and important events. 


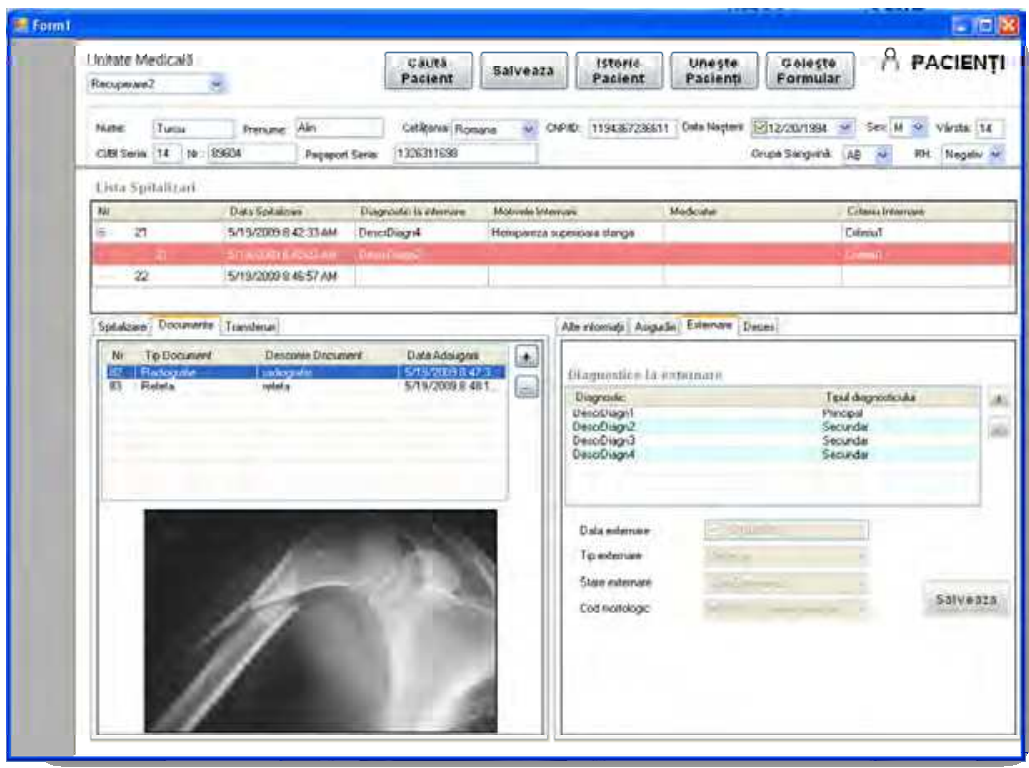

Fig. 8. Patient Demographic module

\section{Conclusions}

Software applications used in medical centers must be modular and scalable in order to meet the needs of the beneficiaries. To ensure the proper integration of various software modules it is necessary to use HL7 and DICOM standards which provides a set of rules and algorithms specific tothe medical field.

\section{References}

NEAC (2008) DICOM standard, http:/ / medical.nema.org/

HL7 (2008) HL7 standard, http:/ / www.hl7.org

Oleg S. Pianykh (2008). Digital Imaging and Communications in Medicine (DICOM), Springer-Verlag Berlin, page numbers (3-7, 301-309), Heidelberg

HIMSS and RSNA, Integrating the Healthcare Enterprise, Revision 5.3 August 15, 2005

Alin Cordos, Mihaela Hedesiu, Bogdan Feldrihan, Marius Rotariu, Transferring medical records to CD/DVD using DICOM standard, 2008 IEEE International Conference on Automation, Quality and Testing, Robotics AQTR 2008, Cluj-Napoca, Romania, May 2008, Poster Session, page numbers (275 - 279)

Cordos, A. Vlaicu, Security implementation in medical network communication, Cost 276/2004, 2004, Ankara, Turkey 


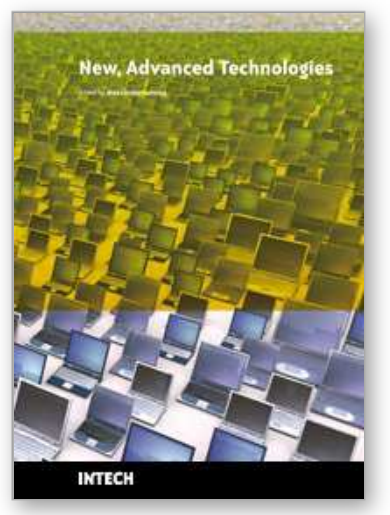

\author{
New Advanced Technologies \\ Edited by Aleksandar Lazinica
}

ISBN 978-953-307-067-4

Hard cover, 350 pages

Publisher InTech

Published online 01, March, 2010

Published in print edition March, 2010

This book collects original and innovative research studies concerning advanced technologies in a very wide range of applications. The book is compiled of 22 chapters written by researchers from different areas and different parts of the world. The book will therefore have an international readership of a wide spectrum.

\title{
How to reference
}

In order to correctly reference this scholarly work, feel free to copy and paste the following:

Orza Bogdan, Cordos Alin, Vlaicu Aurel and Meza Serban (2010). Integrated Medical System Using DICOM and HL7 Standards, New Advanced Technologies, Aleksandar Lazinica (Ed.), ISBN: 978-953-307-067-4, InTech, Available from: http://www.intechopen.com/books/new-advanced-technologies/integrated-medicalsystem-using-dicom-and-hl7-standards

\section{INTECH}

open science | open minds

\section{InTech Europe}

University Campus STeP Ri

Slavka Krautzeka 83/A

51000 Rijeka, Croatia

Phone: +385 (51) 770447

Fax: +385 (51) 686166

www.intechopen.com

\section{InTech China}

Unit 405, Office Block, Hotel Equatorial Shanghai

No.65, Yan An Road (West), Shanghai, 200040, China

中国上海市延安西路65号上海国际贵都大饭店办公楼 405 单元

Phone: +86-21-62489820

Fax: $+86-21-62489821$ 
(C) 2010 The Author(s). Licensee IntechOpen. This chapter is distributed under the terms of the Creative Commons Attribution-NonCommercialShareAlike-3.0 License, which permits use, distribution and reproduction for non-commercial purposes, provided the original is properly cited and derivative works building on this content are distributed under the same license. 